\title{
Technological considerations for 4D printing: an overview
}

\author{
Eujin Pei ${ }^{1}$ (1) Giselle Hsiang Loh $^{1}$
}

Received: 26 December 2017 / Accepted: 2 May 2018 / Published online: 22 May 2018

(c) The Author(s) 2018

\begin{abstract}
4D printing utilizes additive manufacturing methods to produce stimulus-responsive components that can change its shape from one to another when subject to appropriate stimuli. The use of 4D printing technology is expected to significantly become more widespread with more applications across bio-medical, aerospace, and defence industries. This paper discusses emerging applications for $4 \mathrm{D}$ printing and suitable stimulus-responsive materials for $4 \mathrm{D}$ printing. In terms of designing for 4D printing, aspects of the shape memory effect (SME) including one-way SMEs, two-way SMEs and three-way SMEs are presented. Materials and structures in the form of homogenous compositions and heterogeneous compositions are discussed, as well as different types of shape-shifting behaviours such as self-folding, self-assemblies, and self-dis-assemblies. Finally, current software and examples are presented together with the existing limitations that need to be overcome to achieve widespread adoption of $4 \mathrm{D}$ printing.
\end{abstract}

Keywords 4D printing $\cdot$ Four-dimensional printing $\cdot$ Smart materials $\cdot$ Shape-changing effect $\cdot$ Shape memory effect $\cdot$ Selfassembly

\section{4D printing: a radical shift in additive manufacturing}

Current research has seen a radical shift in additive manufacturing ( $\mathrm{AM}$ ) research heading towards 4D printing, introducing Time as the fourth dimension. 4D printing is defined as the use of AM to produce stimuli-responsive parts that can shape change or function change from one form to another when subject to appropriate stimuli without reliance on robotics or electromechanical devices [1]. $4 \mathrm{D}$ printed component is time-dependent yet predictable [2]. The transformation is due to the properties of stimulusresponsive materials that can react to fluctuations in the external environment and generate a response in geometry change (Fig. 1).

The concept of $4 \mathrm{D}$ printing relies predominantly on five factors-the AM process, types of stimulus-responsive material, stimuli, interaction mechanism, and mathematical modelling [2]. The use of AM processes enables freeform objects to be produced directly from digital information

Eujin Pei

eujinpei1@gmail.com

1 Institute of Materials and Manufacturing, Brunel University London, Kingston Lane, Uxbridge UB8 3PH, UK without the need for intermediate shaping tools. Most AM processes can support $4 \mathrm{D}$ printing as long as the selected stimulus-responsive material is supported by or compatible with the printer. For a single multi-material print, a 3D printer capable of multiple-material printing is required to combine two or more materials to produce heterogeneous composition. AM technologies that are capable of multimaterial printing include, but not limited to, the PolyJet Stratasys Connex 3 Objet printer, FDM the RoVa3D 5 Nozzle 3D printer from ORD Solutions, Original Prusa i3 multimaterial upgrade $[3,4]$.

Stimulus-responsive material, often known as smart materials or programmable materials, is highly dynamic in form and functions [5]. The type of stimuli-responsive materials is the key element to grant the capability of selftransformation and determines the type of stimuli needed to trigger the change in property and the functionality of the component in $4 \mathrm{D}$ printing. The properties of stimuliresponsive materials permit the phenomena of coupling or conversion of energy between various physical domains; for example, converting thermal energy into mechanical work. This coupling of energy can be direct or indirect. Direct energy coupling refers to mechanical response due to fieldinduced eigenstrain in the stimulus-responsive materials, whereas indirect is mechanical response due to field-induced 


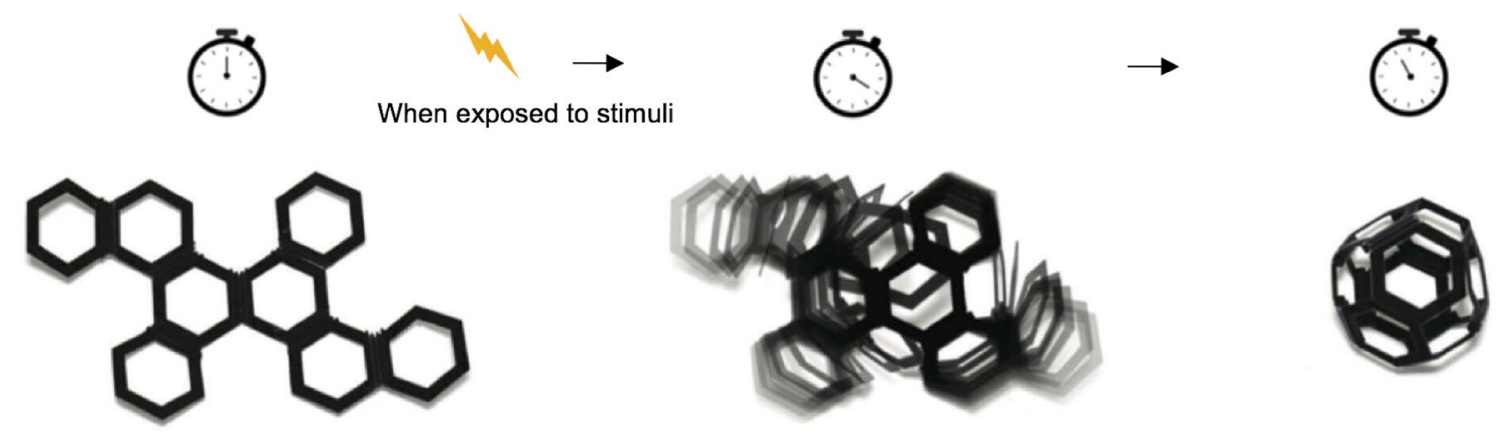

Fig. $14 \mathrm{D}$ printed self-folding truncated octahedron demonstrating the "transformation over time" when submerging in water [1]

change in stiffness or other properties. The types of stimulus-responsive materials capable of change in physical properties can be classified into shape-change material and shape memory material. Shape-change material possessed stimulus-induced behaviour known as shape-change effect (SCE). Shape-change material transforms instantly and spontaneously in response to its stimulus, and returns to its original or permanent shape when the stimulus is removed. Shape memory polymers have the ability to memorize and recover to their trained shape from a temporary shape when stimulus is applied, known as shape memory effect (SME). The complete shape memory cycle will be discussed in Sect. 4.

The types of stimuli can be categorised into physical, chemical, and biological energy. Physical stimuli include temperature, moisture, light, ultraviolet light, magnetic energy, and electricity. Chemical stimuli include chemicals, oxidant, and reductants and based on ionic strength and $\mathrm{pH}$ level, whereas biological stimuli include substances such as the presence of glucose and enzymes. Most experimental studies focused on the use of water [6-9], heat [10-15], a combination of heat and water $[16,17]$ as well as a combination of heat and light [18]. The primary shape-shifting response or output are fold, curl, twist, expansion, and contraction [1, 19, 20].

Not all stimulus-responsive components can undergo intended transformation when exposed to optimum stimulus. It requires a set interaction mechanism to plan out the sequence of shape-shifting behaviours when triggered by the stimulus under an appropriate amount of time. For the phase fixation of shape memory materials, mechanical loading methods or physical mechanism manipulation techniques have to be designed to deform and program the structure at a temporary shape (Fig. 4).

Mathematical modelling is necessary to plan out the required amount of time and sequence of stimulus to act on the stimulus-responsive component. For single multi-material 4D print, this process further involves design of material orientation and distribution, the calculation of different expansion or contraction rates of each stimulus-responsive material to function comprehensively in an effective selftransform system. The mathematical modelling is usually undertaken in conjunction with geometric programming using computer-aided-design (CAD) and finite-element analysis (FEA).

\section{Applications for 4D printing}

The use of 4D printing supports product design freedom with a little additional cost for complexity [21]. For example, products can be designed to be adaptable and tuned to free energy in the environment such as the use of moisture, temperature, pressure, altitude, or sound as potential stimuli. The growing interest in 4D printing has been kindled by research in responsive structures for soft robotics and printable actuators [17] for use in medical devices, smart textiles, defence, and aerospace. For self-assembly, AM parts are no longer constrained by the size of the print bed. Parts can be programmable for postfabrication, whereby parts can be self-assembled to reduce the volume for the initial storage, paving the way for flat-packed cargo. These responsive structures can be printed in a simple geometric form before transforming into a larger and more complex design to reduce the overall print time and manufacturing complexity. Individual parts can be printed using smaller AM systems and later self-assembled into a larger component. One example proposed by Zhou [22] is the transfer of nano-scale parts inside the human body which will undergo self-assembly at a desired location for a specific medical purpose. Other applications include space exploration in which parts can assemble at a predetermined environment without the need for an external power source or human intervention. For soft robotics, 4D printing is moving towards true material robotics by minimising and removing the need for expensive, error-prone, and complex electromechanical devices such as motors, sensors, and electronics as the conventional robotic mechanisms are often bulky, energy consuming, and difficult to assemble [23]. 4D printed 
structures can also be designed to self-repair. Some possible applications include flexible self-healing pipes by Campbell [24] and self-healing hydrogels [25]. The use of 4D printing technology is expected to significantly become more widespread with more applications across different sectors [3]. Frost and Sullivan [26] predicted that applications used by $4 \mathrm{D}$ printing technology in the future could include adaptable sensors, for bio-printing artificial organs and for space and automotive parts.

\section{Smart materials for 4D printing}

Stimuli-responsive materials are capable of transformation when there is a change in the environment and this coupling effect or interaction can result in a mechanical response due to field-induced eigenstrain (a deformation produced without external forces such as thermal expansion or phase change) in the smart material, or resulting in an indirect mechanical response due to a field-induced change in stiffness. The transformational characteristics of smart materials include self-sensing, responsiveness, shape memory, self-repair, self-adaptability, and multifunctionality. Smart materials can be classified as shape memory materials and shape-changing materials. Shape memory materials have the ability to recover to their original shape from a temporary shape when stimuli are applied and this is known as the shape memory effect (SME) [27]. Shape memory materials (SMMs) include shape memory alloys (SMAs), shape memory polymers (SMPs), shape memory gels (SMGs), shape memory ceramics (SMCs), and other shape memory hybrid (SMHs) materials [28]. Shape-changing materials are materials that possess stimulus-induced behaviour, known as the shape-change effect (SCE). They morph in response to the stimuli and may return to its permanent shape when the stimuli are removed. Zhou [29] explained that the type of transformation is usually limited to simple affine alterations such as linear volume expansion (stretching) or contraction (shrinking). Homogeneous expansion or contraction (shrinking) in all directions does not lead to a change of geometry. In contrast, inhomogeneous expansion or shrinking will lead to induced surface topography such as buckling, folding, and bending. One of the key challenges in the field of $4 \mathrm{D}$ printing research is to be able to visualize and communicate the material properties physically and digitally (such as by means of individual voxels); and to be able to successfully program the parts so as to achieve a desired transformational change at a specific location.

Shape memory polymers (SMPs) are more popularly used for 4D printing and they will be described in further detail in this paper. For example, SMPs and hydrogels are preferred over the use of shape memory alloys (SMAs) as SMPs have a wide range of glass transition temperatures from -70 to $100{ }^{\circ} \mathrm{C}$, allowing their stiffness to be tailored [30]. SMPs have the potential to achieve a shape recovery property up to $400 \%$ of plastic strain, whereas SMAs are around 7-8\%. SMAs are regarded disadvantageous due to complex manufacturing, higher costs, toxic, and with limited recovery (Table 1). SMPs are predominantly polymeric materials that have an ability to revert back to its preprogrammed shape from a deformed (temporary) configuration when exposed to stimuli [21]. While activated through ambient energy, SMPs exhibit a radical change from a rigid polymer to an elastic state, and back to a rigid state again. Between the glassy and rubbery states of SMPs, large reversible changes of elastic modulus can be observed as high as 500 times. SMPs have high elastic deformation, with a strain up to more than $200 \%$ for most of the materials and they have low density and can be biodegradable as well as biocompatible for medical applications. SMPs have low thermal conductivity compared to SMAs which may be useful if shape memory foams are to be used as insulation materials [31]. The challenge for multimaterial 4DP is to identify and use materials that are strong and malleable in the presence of stimuli. Ideally, the material should also exhibit different behaviour in the presence of different stimuli, and exhibiting a plurality of functions [18].

The potential stimulus-responsive polymeric materials for 4D printing include polylactide (PLA), thermoplastic polyurethane (TPU), and UV-cured thermoset polymers such as VeroWhite Plus RG835 used in material jetting [22].
Table 1 Comparison between SMPs and SMAs [31]

\begin{tabular}{lll}
\hline Property & Shape memory polymers & Shape memory alloys \\
\hline Density $\left(\mathrm{g} / \mathrm{cm}^{3}\right)$ & $0.9-1.2$ & $6-8$ \\
Extent of deformation & Up to $800 \%$ & $<8 \%$ \\
Required stress for deformation $(\mathrm{Mpa})$ & $1-3$ & $50-200$ \\
Stress generated upon recovery $(\mathrm{MPa})$ & $1-3$ & $150-300$ \\
Transition temperature $\left({ }^{\circ} \mathrm{C}\right)$ & -10 to 100 & -10 to 100 \\
Recovery speed & $1 \mathrm{~s} \mathrm{to} \mathrm{min}$ & Less than $1 \mathrm{~s}$ \\
Processing condition & $<200{ }^{\circ} \mathrm{C} ;$ low pressure & $>1000{ }^{\circ} \mathrm{C} ;$ high pressure \\
Cost & $<10 / \mathrm{lb}(£ 7.5 / \mathrm{lb})$ & Approx. $\$ 250 / \mathrm{lb}(£ 189 / \mathrm{lb})$ \\
\hline
\end{tabular}




\section{The 4D printing shape memory effect}

The main characteristic of shape memory materials (SMMs) is the ability to recover to their programmed shape from a temporary shape when stimulus is applied. This is known as the shape memory effect (SME) [27]. SMMs require two processes to form a complete shape memory cycle [32]. The first step is to deform the material into a temporary shape through the "programming process" (Fig. 4), followed by the "shape recovery process". SMMs will remain constant in its temporary shape until the right optimum stimulus is applied to trigger the shape recovery process. The rapidity of shape change from a temporary shape depends on the responsiveness of the material and the physical design of the geometrical part. The network elasticity of the SMM determines the "memory" of one or more shapes. The two significant factors that determine the shape memory effect of SMMs are the strain recovery rate $\left(R_{\mathrm{r}}\right)$ and the strain fixity rate $\left(R_{\mathrm{f}}\right)$. The strain recovery rate $\left(R_{\mathrm{r}}\right)$ refers to the ability of the material to memorize its permanent shape, whereas the strain fixity rate $\left(R_{\mathrm{f}}\right)$ refers to the ability of the switching segments within the mechanical deformation. Both $R_{\mathrm{r}}$ and $R_{\mathrm{f}}$ have to add up to $100 \%$ to be measured as an effective SMP. The calculation for strain recovery and fixity rate is made up of $R_{\mathrm{r}}=100 \% \times\left(\varepsilon-\varepsilon_{\mathrm{rec}}\right) / \varepsilon$ and $R_{\mathrm{f}}=100 \% \times \varepsilon / \varepsilon_{\text {load }}$; whereby $\varepsilon=$ fixed strain after cooling and unloading; $\varepsilon_{\text {rec }}=$ strain after recovery; and $\varepsilon_{\text {load }}=$ maximum strain under load [33].
Fig. 2 One-way shape memory effect

Fig. 3 Reprogramming procedure for a one-way shape memory effect

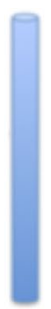

(A)

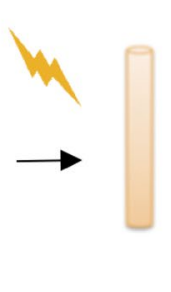

(B)

\section{Reprogramming required}

Programming process

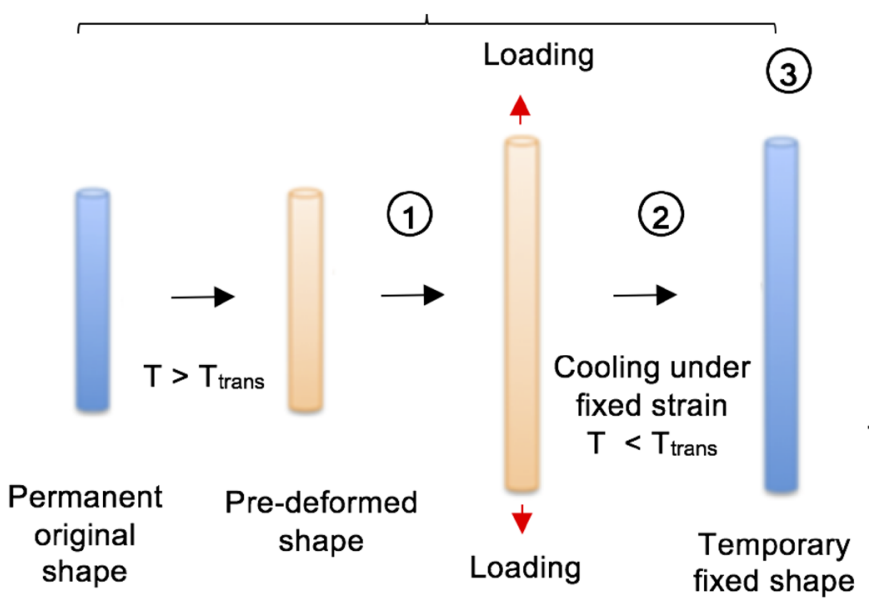

(B)
(A)

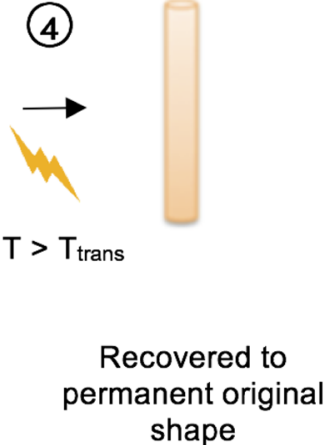

(B)
Fig. 4 Two-way shape memory effect [30]

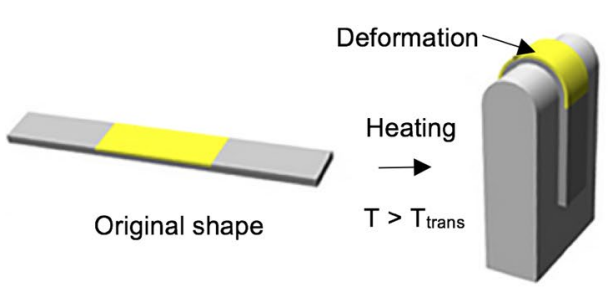

(A)

(B)
: Stimulus 
This paper will study on the shape memory effect (SME) of shape memory polymers (SMPs).

\subsection{One-way shape memory effect}

The majority of SMPs have a one-way shape memory effect which is irreversible. When an external stimulus is applied, the deformation (temporary) shape will become a permanent shape. A programming step (Fig. 2) is needed for the object to return back to its temporary shape.

Figure 2 describes the process of the one-way shape memory effect where the SMP changes from its temporary shape (A) back to the permanent original shape (B) under an applied stimulus. In the programming process, the SMP is first heated above transition temperature to soften the material, so that a deformation force (e.g., loading) can be applied to the original shape. The predeformed shape is cooled under the load to a fixed temporary shape. When the unloaded fixed temporary shape is exposed to stimuli, in this case is heat, the original shape (B) is recovered (Fig. 3). In terms of heating, Behl [34] explained that there are various types of thermal transition temperatures that are associated with switching domains. It includes the melting transition $\left(T_{\text {trans }}=T_{\mathrm{m}}\right)$, liquid crystalline transition $\left(T_{\text {trans }}=T_{\mathrm{LC}}\right)$, or glass transition $\left(T_{\text {trans }}=T_{\mathrm{g}}\right)$. However, most melting and liquid crystalline phase transitions have relatively small temperatures. For the shape memory effect, the glass transition $\left(T_{\mathrm{g}}\right)$ temperature is usually considered.

1. deforming the structure (B) at temperature above transition temperature ( $\left.T>T_{\text {trans }}\right)$ by mechanical loading;

2. phase fixation in which temperature is lowered below transition temperature $\left(T<T_{\text {trans }}\right)$ with the mechanical loading remained;

3. phase transformation in which the load is removed and desired temporary fixed shape (A) is achieved;
4. shape recovery from (A) to (B) by reheating the structure up to transition temperature ( $\left.T \geq T_{\text {trans }}\right)$.

\subsection{Two-way shape memory effect}

SMP with two-way shape memory effect has the ability to remember two different shapes when exposed to stimuli. The material can change from a temporary shape back to its permanent shape (Fig. 4) and the change is reversible. Zhou [35] emphasised that this behaviour is neither mechanically nor structurally constrained, thereby allowing for multiple switching between encoded shapes without applying any external force. The two-way SME can be found in liquid crystalline elastomers and photo-actuated deformation polymers [36]. Chen et al. [37] successfully demonstrated the two-way shape memory behaviour using a polymer laminate prepared from a $1.0 \mathrm{~mm}$-thick active layer of PHAG5000 polyurethane-based shape memory with a $1.0 \mathrm{~mm}$-thick substrate of PBAG600-based polyurethane. The effect was observed by bending upon heating from 25 to $60^{\circ} \mathrm{C}$ and reverse bending upon cooling from 60 to $25^{\circ} \mathrm{C}$.

\subsection{Three-way shape memory effect}

According to Erkeçoğlu [30], the main difference between a one-way and three-way shape memory effect is that the three-way shape memory effect has one intermediate shape between its original and temporary shapes. If there is more than one intermediate shape, then this is also known as a "multiple shape memory effect", achieved by combining multiple two-way shape memory polymers with different glass transition temperatures [38]; or by heating a programmed shape memory polymer first above the glass transition temperature and then above the melting transition temperature of the switching segment [39]. The threeway shape memory effect is shown in Fig. 5, whereby there are two different thermal transition temperatures $-T_{\text {low, } 1}$
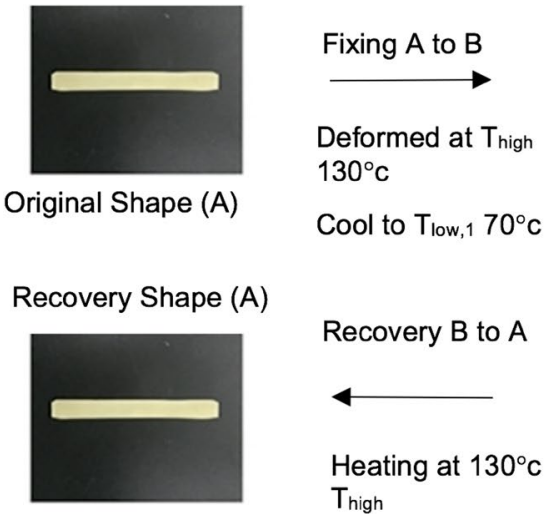

Recovery B to A

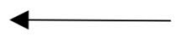

Heating at $130^{\circ} \mathrm{C}$ Thigh

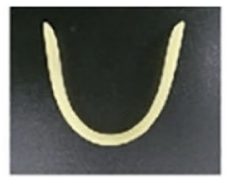

Temporary Shape (B)

Recovery Shape (B)

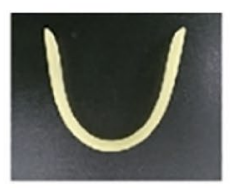

Fixing $B$ to $C$
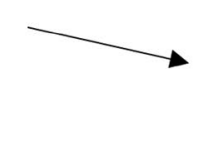

Recovery $\mathrm{C}$ to $\mathrm{B}$

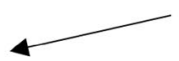

Heating to $\mathrm{T}_{\text {low }, 1} 70^{\circ} \mathrm{C}$
Deformed at $\mathrm{T}_{\text {low, } 1} 70^{\circ} \mathrm{C}$

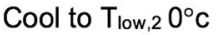

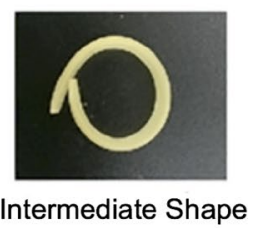

(C)

Fig. 5 Three-way shape memory effect [40] 
$\left(70{ }^{\circ} \mathrm{C}\right)$ and $T_{\text {low, } 2}\left(0^{\circ} \mathrm{C}\right)$. This is attributed to the two segregated crystalline domains in the original shape. $\mathrm{Li}$ [40] listed several methods to manage triple shape memory effects such as by blending, grafting, and blocking copolymers, SMP hybrids, or other polymer laminates.

A material with dual-SME is able to achieve one permanent shape and one temporary shape, whereas a tripleSME material can achieve one permanent shape and two temporary shapes. The dual shape mechanism (Table 2) is achieved by assembling two components in the form of hard and soft segments within a single matrix. This single component is able to transition into a pliable state upon reaching the glass transition $\left(T_{\mathrm{g}}\right)$ temperature and the original shape can be recovered. A typical example is to utilize the glass transition of the polymer. Above the $T_{\mathrm{g}}$, a polymer is in the rubbery state and soft and can be easily deformed. Upon cooling to below $T_{\mathrm{g}}$, the polymer is in the glassy state and hard. The original shape may be recovered upon heating to above the $T_{\mathrm{g}}$. The cross-linked chains serve as an element to store the elastic energy which acts as the driving force for shape recovery [32]. The term dual-component mechanism (DCM) is referred when two components are assembled in the form of hard and soft segments within a matrix. The hard segment is elastic within the working temperature and the soft segments become pliable upon heating, and the material deforms at high temperatures [21].

\section{Design of 4D printed parts and material compositions}

To fully capitalize on the use of smart materials, the design of the 4D printed structure and the assignment of materials will require novel tools and methods [41]. Specialized software is needed to design specific folds, creases, and patterns, and to demonstrate the sequence of folding. A longstanding challenge is to find a way to control the sequence in which the structure will fold in a controlled manner [42]. Project Cyborg from Autodesk is a cloud-based meta-platform of design tools capable of programming matter from nanoparticle to human-scale manufacturing. The software can simulate self-assemblies and programmable materials, and specify the optimisation parameters for geometrical transformation, shape constraints, and the folding sequence [6]. Other pattern folding software such as Origamizer can be used to create complex origami shapes through assigning nodes, edges, paths, polygons, vertices, and creases [43]. The software is capable of generating crease patterns that can fold solid geometries into a complex polyhedral model with a designated number of seams. Another example is the E-Origami System developed by the Symbolic Computation Research Group (SCORE) that can study the mathematical aspects of paper folds. This system allows users to fold sheets using algebraic and symbolic methods. It offers two methods of folding, classified as mathematical folds and artistic

Table 2 Differences between a dual-and-triple shape memory effect

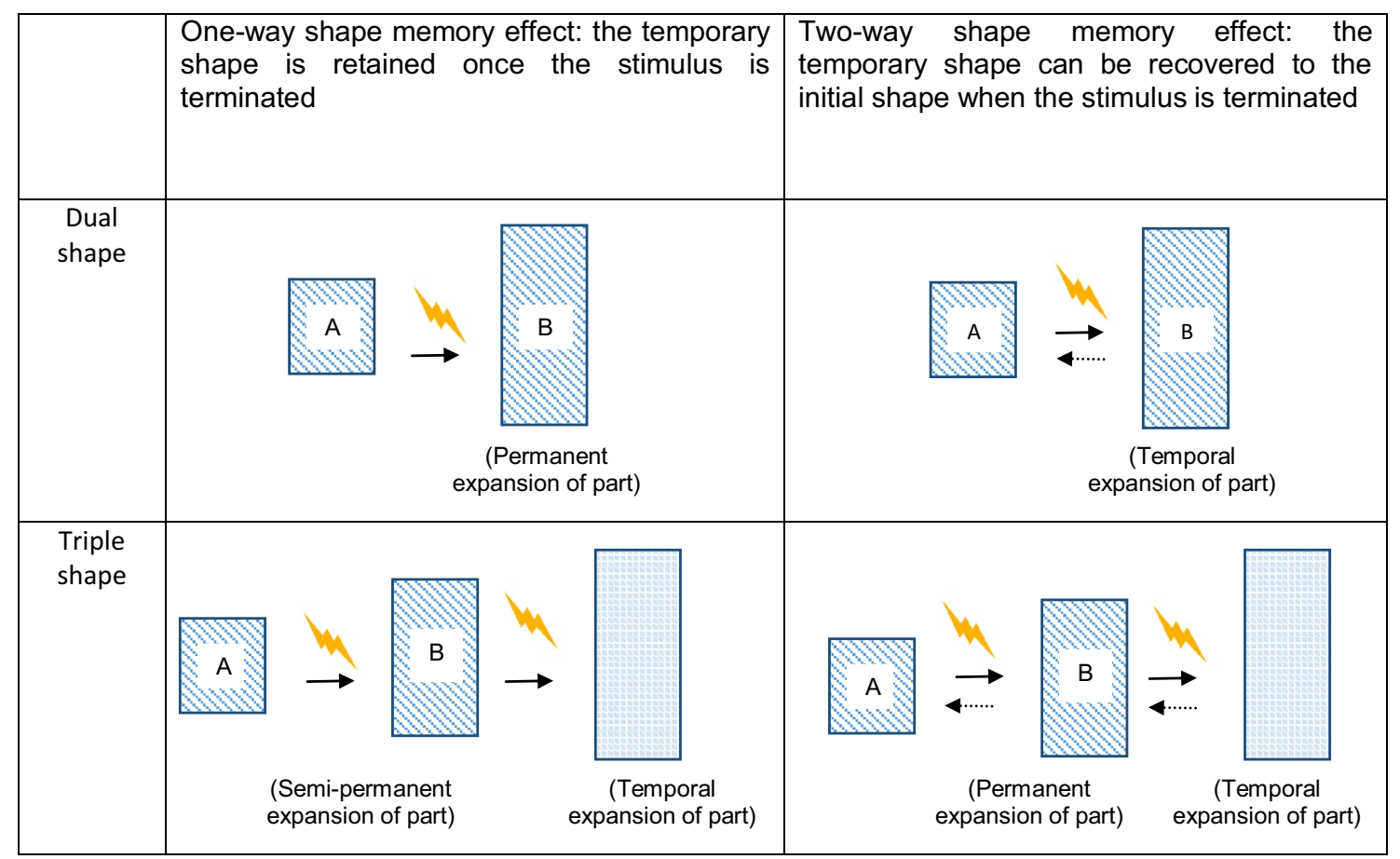


folds. The mathematical method of folding is based on the axiomatic definitions of origami folds; whereas the artistic fold is ideal for the creation of imaginative origami pieces as it can specify relatively straightforward folding features such as whether the fold is a valley or a mountain, which lines are to be folded, and to what angle which they should be folded. Inversely, Akleman et al. established a simulation software to unfold a given convex polygonal mesh into either multiple or one-piece planar sheets [44]. They claimed that the algorithm for single-panel unfolding first divides the initial shape into triangles to guarantee that all faces of the shape are planar. Next, a 'dual mesh' is constructed from the triangulated shape, such that every face becomes a vertex and every original vertex becomes a face. Every vertex of the dual graph is then two-dimensionally thickened into a triangle and every edge of the spanning tree is two-dimensionally thickened into a developable quadrilateral. The material composition of $4 \mathrm{D}$ printed parts can be described as being homogenous (single material) or heterogeneous (multiple materials). A homogeneous composition consists of the use of a single material. Yang [32] demonstrated the concept of a single material 4D printed as a self-tightening mechanism made from PLA (Fig. 6). The shape-shifting effect of a single material can be advanced by adjusting the gradient distribution of the material by strategically controlling the spatial position (e.g., density and porosity) and morphology (e.g., design and arrangement) of the lattice structure across the component.

A heterogeneous $4 \mathrm{D}$ printed part consists of a mixture of two or more materials being printed as a single component. The materials can be a combination of smart or conventional composite materials or using various smart materials that react differently under various stimuli. Kuksenok [18] proposed a stimuli-responsive mechanism that integrated thermo-responsive gels with photo-responsive
Fig. 6 Homogeneous 4D printed staple that demonstrates the tightening function [29]

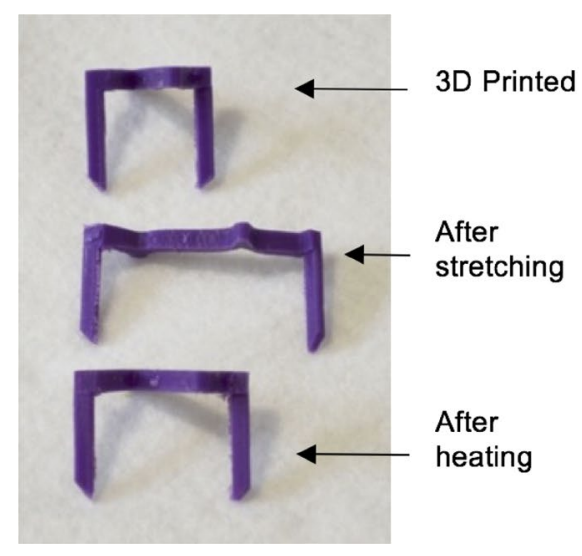

Evolution of the untethered sample under the light

Fig. 7 Heterogeneous stimuliresponsive mechanism integrating thermo-responsive gels with photo-responsive fibre-global and local shrinkage and bending [18]

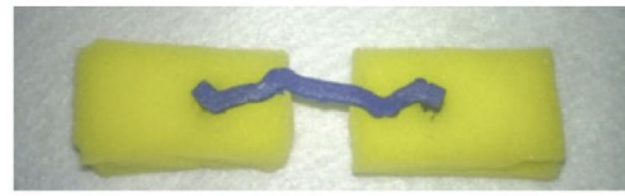

Before shape recovery

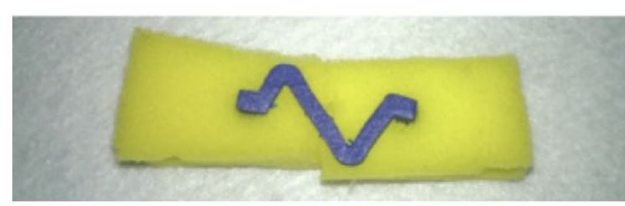

After shape recovery

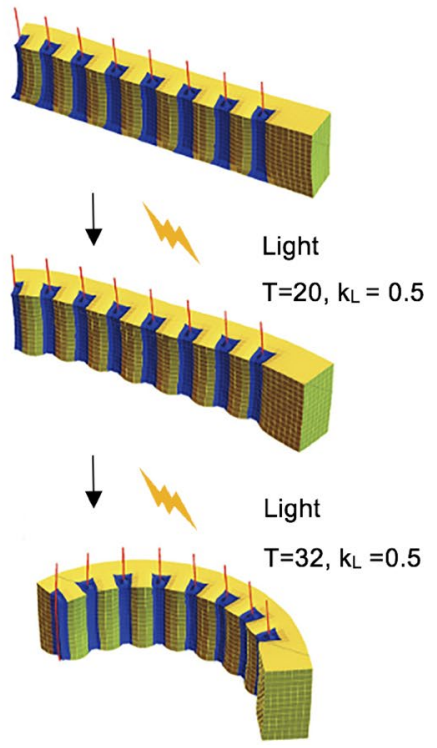

Evolution of the untethered sample in the dark at high temperature.

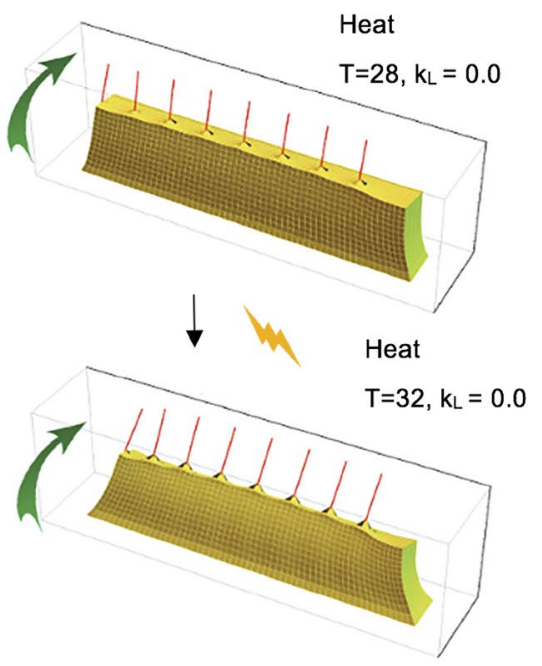



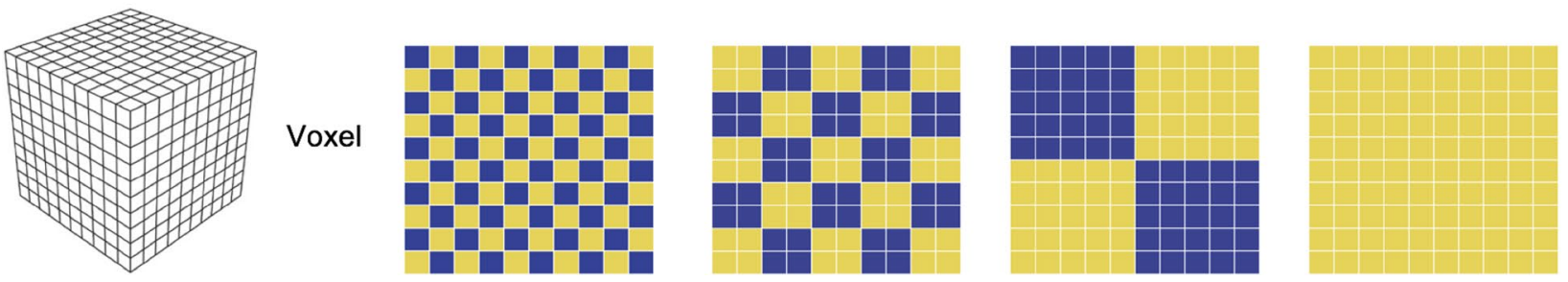

Fig. 8 Uniform distribution of digital materials

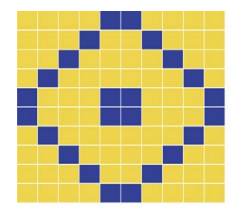

(A)

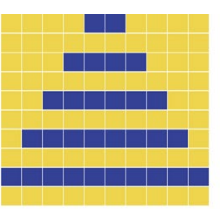

(B)

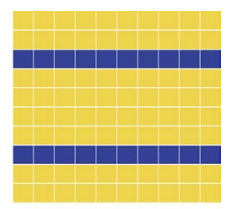

(C)
Fig. 9 a Gradient from centre to edge; $\mathbf{b}$ one-way gradient; and $\mathbf{c}$ patterned gradient

fibres to demonstrate global shrinking and localized bending, whereby the structure has controllable and repeatable dynamic movements when exposed to light and heat (Fig. 7).

Innovate from the conventional multi-material printing, another approach of achieving a heterogeneous composition 4D printed part is using functionally graded additive manufacturing (FGAM). FGAM is a layer-by-layer fabrication process that involves gradationally varying the material organization within a component to achieve an intended function. It can generates dynamically composed gradients or through complex morphology of individual voxels [45, 46]. Voxels are the fundamental building blocks within the $3 \mathrm{D}$ digital space. In this case, the properties and functionality of a 4D printed structure are determined by the spatial arrangement of the voxels, whereby each individual voxel represents a single material with its own properties. Consequently, a collection of voxels with different material information contributes to a multi-material structure. Digital materials function as an extensible modelling and software infrastructure to support the representation and simulation of the material structure across multiple time scales and compositions. It can serve as an enabler to control and simulate the materials [47]. The arrangement of multi-material 4D printed structure using digital materials can be classified as having a uniform distribution (Fig. 8) or having a graded distribution (Fig. 9).

\section{Types of shape transformation effects of 4D printed parts}

Shape-shifting behaviours that researchers have explored tend to focus on simple forms and using a bilayer composite comprising of soft hyper-plastics and SMPs [48]. Such geometric programming involves two or more active materials in which the desire to generate complex and multiple shapes from the same starting material can be achieved by programming the sequence of shape transformation with temporal control. Self-folding and bending structures are more commonly applied in 4D printing. The difference between folding and bending is shown in Fig. 10. A fold is a deformation in which the in-surface distance between any two points in the sheet is preserved without self-intersection [49]. Bending is a global deformation associated with a smoother distributed curvature, whereas folding emphasises on localized deformation with sharp angles in a narrow hinge area [50]. Most shape-shifting behaviour from 2D into 3D is achieved through bending that is caused by expansion or contraction of materials with different magnitudes in different directions $[51,52]$.

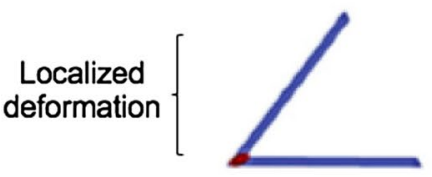

Folding
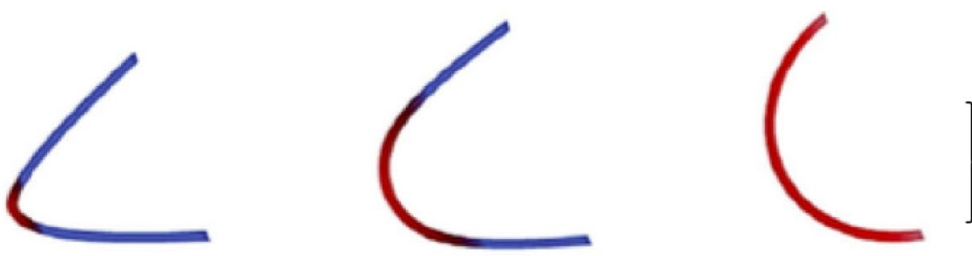

Smoother curvature

Fig. 10 Differences between folding and bending [53] 

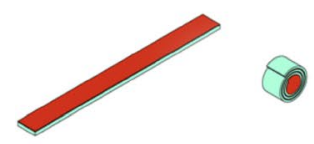

(A) Roll

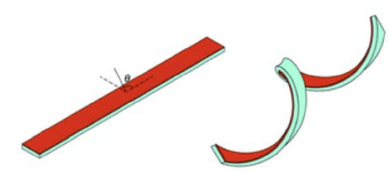

(B) Spiral Twist

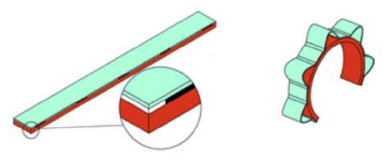

(C) wave-like

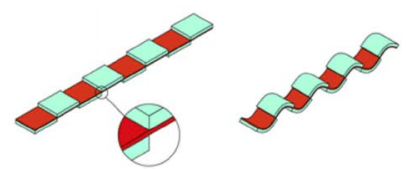

(D) Wrinkled and rolled

Fig. 11 Primary shape transformations of self-folding polymers are roll, spiral twist, wave-like structure, and wrinkled and rolled [48]

Fig. 12 Extensional active fold
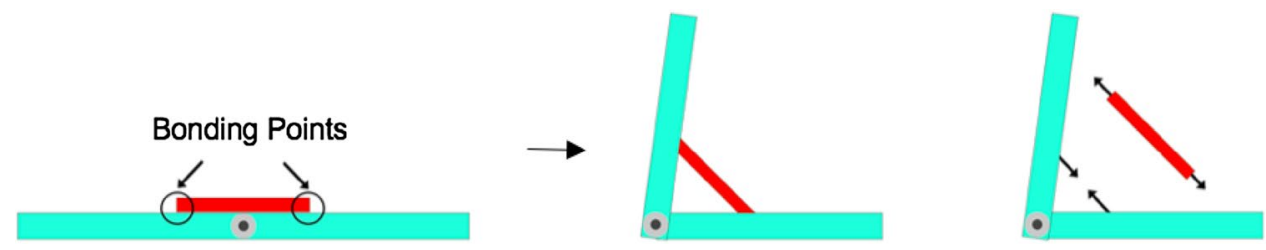

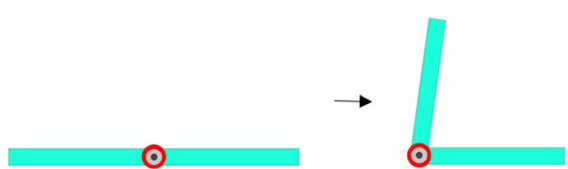

Fig. 13 Torsional active fold

The term self-folding is a class of self-assembly mechanism that uses a shape transformation effect such as folding, curving or rolling from a printed patterned geometry or using thin films that are fabricated in spiral-shaped or cylindrical tubes (Fig. 11) [43, 54]. The self-folding concept can be classed as those with hinges or without hinges. Significant design parameters or drivers that need to be considered while designing active self-folding mechanisms include the selection of the folding mechanism, the size of the component and the type of material. It is also important to account for the actuation strain, actuation stress, and the capacity of generating and manipulating the desired field at the chosen location of the bend [43].

Folding mechanisms can be operated using hinge-based active folding mechanisms by means of a extensional fold with a variable length of active rod or a spring connected to the two faces joined by the hinge (Fig. 12); a torsional fold that uses an active torsional element at the hinge (Fig. 13); or a flexural fold that uses an active element with a predetermined folded shape (Fig. 14) [43].

Bending can be classified into two categories in terms of a layered structure (Fig. 15) or a functionally graded composition of smart materials (Fig. 16). The thickness and dimensions of the different layers play a critical role in determining the bending degree of the printed component [13]. Most self-folding polymers can undergo considerable
Passive material

Active material 1

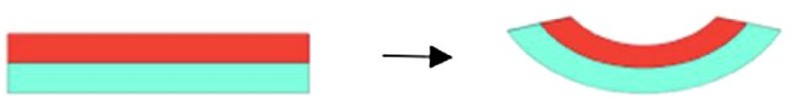

Fig. 15 Conventional multi-material printing to create a bilayer of an active and a passive layer

\section{Active material 1}

\section{Active material 2}

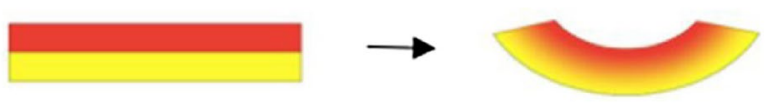

Fig. 16 Functionally graded AM heterogeneous composition of two active materials

and reversible changes of volume, being ideal for systems that require reversible folding [51].

Other researchers have focused on the design of more complex stimuli-responsive mechanisms. For example, Rajiv et al. [8] demonstrated three examples of deformation including linear stretching, folding, and ring stretching through various configurations of multi-material components that would react to water. In the case of linear stretching, the rigid material acts as a scaffold, while the active 
(hydrophilic) layer generates the force for action. This method was achieved by assembling a series of rigid disks with expanding materials in the middle. By adjusting the ratio of expanding materials to the number of rigid disks, the length (and extent) of stretching can be effectively controlled (Fig. 17).

Rajiv et al. [8] also proposed folding that can be achieved by adjusting the distances between the stoppers, the rigid plates of different spacing, and the diameters between the bars to define the angle of fold. This is achieved by printing a layer of hydrophilic material over a layer of rigid material and the disks in the centre act as stoppers to prevent the fold from bending further (Fig. 18).

Finally, they proposed that ring stretching can be achieved by forming a stack of ring-like shapes. Each ring is made up of two layers of material. When the component is submerged in water, the inner layer expands and this causes a deformation of the ring. The stretching length is adjusted by controlling the radius of the ring (Fig. 19).

Origami is the Japanese art of paper folding in which desired shapes are achieved through the sequence of folding from a planar sheet. The folding principles of origami have shed new approaches for manufacturing, assembling, and morphing structures. It involves three steps to design active origami structures for $4 \mathrm{D}$ printing. The first step is to decide the desired shape (magnitude of fold), second is to identify the crease patterns needed, and, finally, testing to ensure that the folding sequence is in the right order to achieve the desired shape (folding kinematics). The creases are defined by their endpoints and the regions within the sheet bound by the creases known as faces (Fig. 20). A 'mountain-valley' feature determines the fold direction of a crease [43].
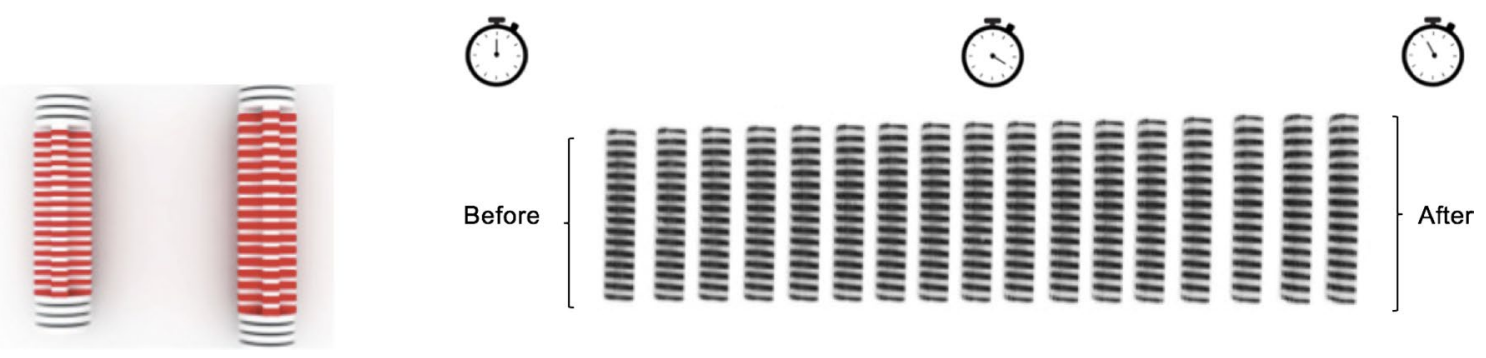

Fig. 17 Linear stretching [8]

Fig. 18 Folding [8]
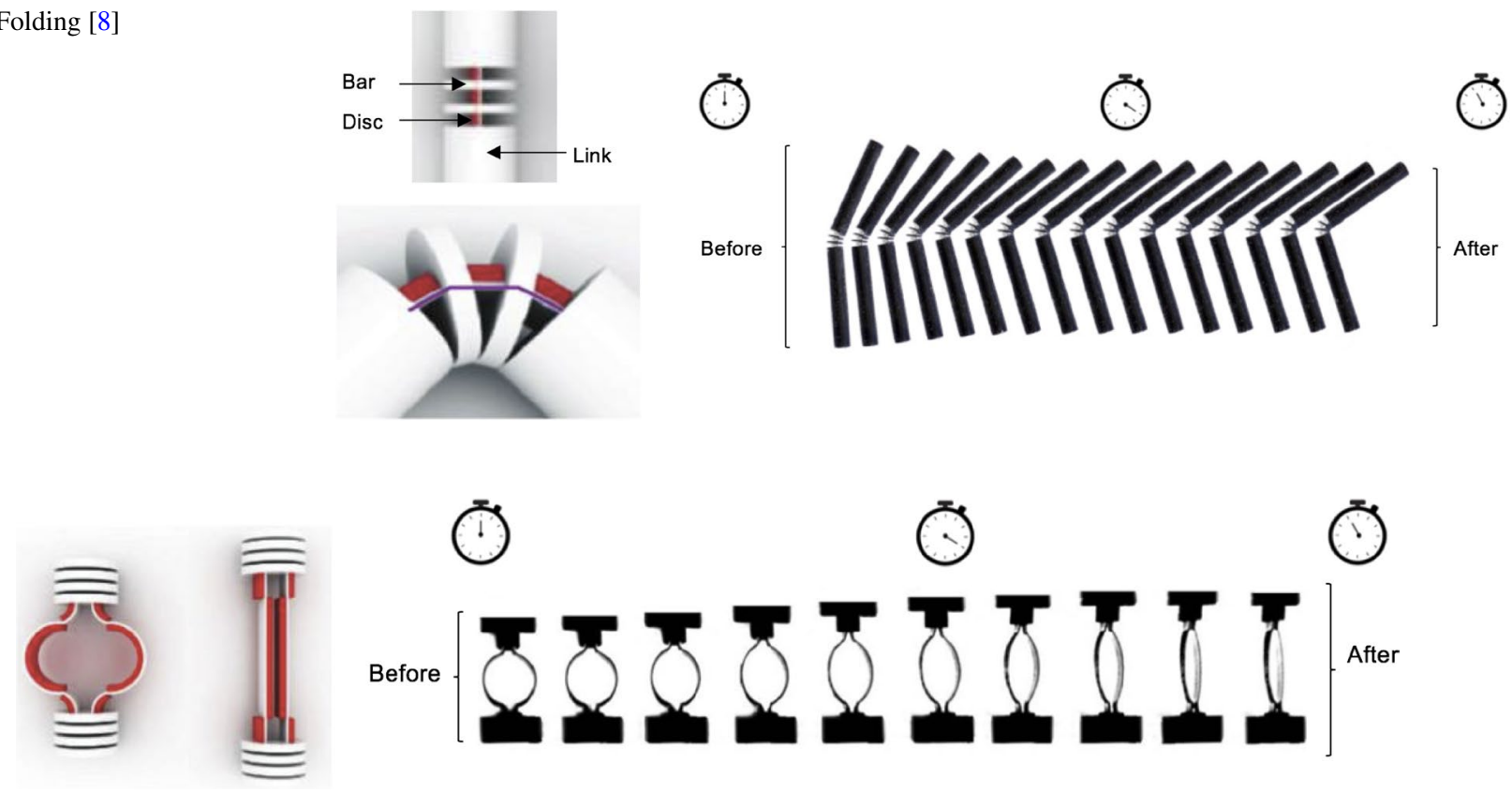

Fig. 19 Ring stretching [8] 


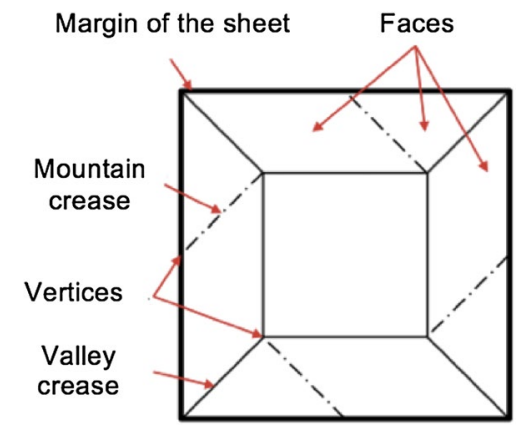

Fig. 20 Schematic of a crease pattern

\section{Conclusion}

Researchers have begun to explore the possibilities of 4D printing as an emerging technology as additive manufacturing technologies mature in parallel to our growing knowledge of smart materials, available stimuli, mathematical modelling, and geometric programming. This paper has provided an overview of $4 \mathrm{D}$ printing, covering the material, technological, and design considerations that should be considered when developing components for 4D printing. The paper discusses emerging applications for $4 \mathrm{D}$ printing and the use of smart materials for $4 \mathrm{D}$ printing. Aspects of the shape memory effect (SME), one-way SMEs, two-way SMEs, and three-way SMEs are presented as well as potential materials and structures in the form of homogenous compositions and heterogeneous compositions are discussed. For 4D printing to become commercially achievable, further research needs to be carried out. For example, computer-aided design software need to be able to visualize the properties of smart materials in conjunction with the use of multi-scale physics simulation. Fabrication systems have to be sufficiently reliable to ensure that the spacing between the voxels that represent the materials are suitable and reproducible. Finally, new metrology equipment or other forms of measurements may need to be developed to ensure the quality of parts.

Future work will focus on realising 4D printed parts that eliminate the need for mechanical loading. For example, recent work by Mao [13] produced a multi-material layered composite with a reversible shape-changing effect. Each material reacts differently to the stimuli, thereby switching behaviours between two stable and stiff configurations without the need for mechanical loading. The controlled spatial distribution and temporal arrangements also determine the geometrical and temporal shape-change characteristics, mechanical stiffness, and load-carrying capacity for each configuration. The lower layer is made up of a hydrogel sandwiched between two columns of elastomers, while the top is placed with a layer of thermo-responsive SMP. Small holes are made within the elastomer layer to permit the flow of water, in which the design restricts the swelling of the hydrogel in the $Z$ direction, allowing the shape change to only occur in the $X-Y$ direction. In addition, the difference in stiffness between the elastomer and the SMP means that the strip bends inwards. More importantly, the thermo-sensitive SMP is carefully designed in such a way to regulate the sequence of time for the shape change to take place. Other novel work includes parts that are capable of self-assembly and disassembly. For example, $\mathrm{Wu}$ [12] proposed a multipolymer 3D printed trestle design with four equal active composite strips connected to the center (Fig. 21).

The 3D printed structure is programmed with load of $8 \%$ strain when stretched at $70{ }^{\circ} \mathrm{C}$. The temperature is reduced to $0{ }^{\circ} \mathrm{C}$ and the load is removed. After releasing at a low temperature, the $4 \mathrm{D}$ printed structure bends with a slight curvature. The strips fold and the trestle stands up from the flat shape as the temperature increases. The trestle goes back to its flat shape when it reaches $70{ }^{\circ} \mathrm{C}$ (Fig. 22). Shapeshifting behaviour can undergo more complex actuations; however, the current information regarding the folding deformation is still relatively limited [2]. Reversibility of the transformation has been relatively unexplored, especially for applications that require cycles of folding and unfolding, or wetting and drying. Comprehensive tests are required to

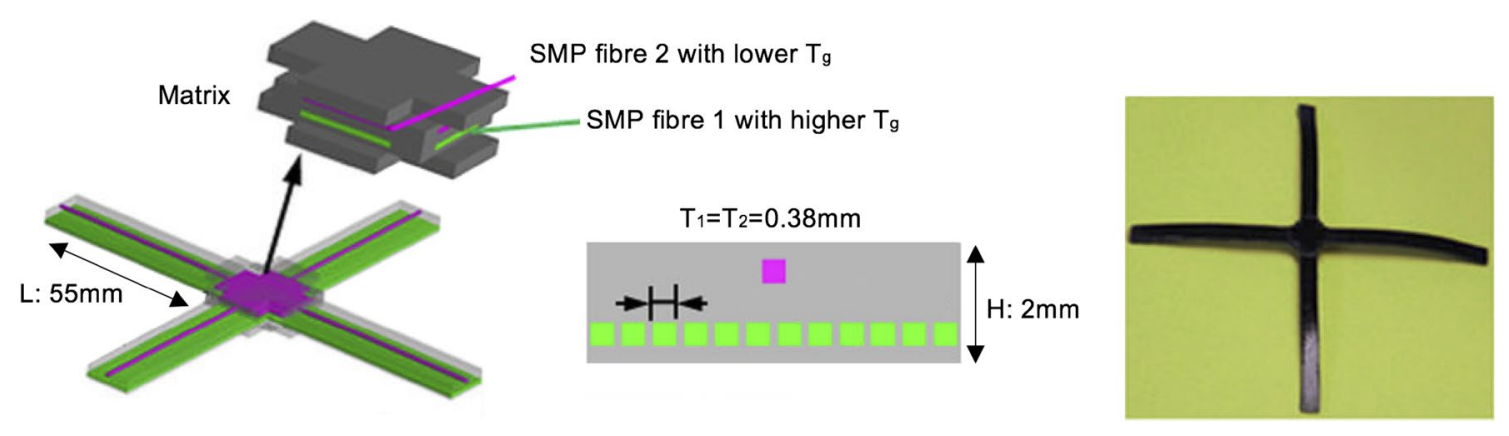

Fig. 21 Design of the trestle, cross section of the composites strips, and the 3D printed part [12] 

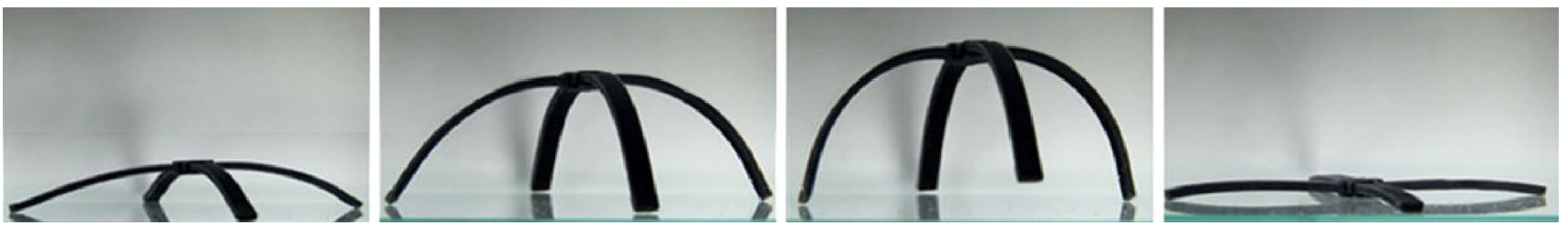

Fig. 22 Deformation behaviour of the structure [12]

fully understand the complete lifespan and the degradation of the materials. Future research will need to investigate how we can generate, store, and use passive and abundant energy sources to activate those shape memory or stimuliresponsive materials.

\section{Compliance with ethical standards}

Conflict of interest On behalf of all authors, the corresponding author states that there is no conflict of interest in this work.

Open Access This article is distributed under the terms of the Creative Commons Attribution 4.0 International License (http://creativeco mmons.org/licenses/by/4.0/), which permits unrestricted use, distribution, and reproduction in any medium, provided you give appropriate credit to the original author(s) and the source, provide a link to the Creative Commons license, and indicate if changes were made.

\section{References}

1. Tibbits S (2013) 4D printing. MIT self-assembly lab and Stratasys. http://www.selfassemblylab.net/4DPrinting.php. Accessed 24 Nov 2017

2. Momeni F, Hassani. N SMM, Liu X, Ni J (2017) A review of 4D printing. Mater Des 12:42-79

3. Khan IA, Liew CS, Loy YY, Lu W, Myint PN, Soh KBA (2015) 4D printing with smart materials. https://www.slideshare.net/ Funk98/4d-printing-with-smart-materials. Accessed 18 Feb 2018

4. Prusa Research (2018) Printer upgrades. https://shop.prusa 3d.com/en/31-printer-upgrades. Accessed 29 Apr 2018

5. Tibbits $S$ (2017) Self-assembly lab: experiments in programming matter. Taylor \& Francis, Abingdon (ISBN 1317437020, $\mathbf{9 7 8 1 3 1 7 4 3 7 0 2 4 \$ 4 )}$

6. Tibbits S (2014) 4D printing: multi-material shape change. Archit Des 84(1):116-121

7. Tibbits S, McKnelly C, Olguin C, Dikovsky D, Hirsch S (2014) 4D printing and universal transformation. http://papers.cumincad. org/data/works/att/acadia14_539.content.pdf. Accessed 18 Dec 2017

8. Raviv D, Zhao W, McKnelly C, Papadopoulou A, Kadambi A, Shi B, Hirsch S, Dikovsky D, Zyracki M, Olguin C (2014) Active printed materials for complex self-evolving deformations. Sci Rep 18(4):7422

9. Jamal M, Kadam SS, Xiao R, Jivan F, Onn TM, Fernandes R, Nguyen TD, Gracias DH (2013) Bio-origami hydrogel scaffolds composed of photocrosslinked PEG bilayers. Adv Healthc Mater 2(8):1142-1150
10. Ge Q, Qi HJ, Dunn ML (2013) Active materials by four-dimension printing. Appl Phys Lett 103:131901

11. Ge Q, Dunn CK, Qi HJ, Dunn ML (2014) Active origami by 4D printing. Smart Mater Struct 23(9):094007

12. Wu J, Yuan C, Ding Z, Isakov M, Mao Y, Wang T, Dunn ML, Qi HJ (2016) Multi-shape active composites by 3D printing of digital shape memory polymers. Sci Rep. https://doi.org/10.1038/srep2 4224

13. Mao YQ, Ding Z, Yuan C, Ai S, Isakov M, Wu J, Wang T, Dunn ML, Qi J (2016) 3D printed reversible shape changing components with stimuli responsive materials. Sci Rep. https://doi. org/10.1038/srep24761

14. Yu K, Dunn ML, Qi HJ (2015) Digital manufacture of shape changing components. Extreme Mech Lett 4:9-17

15. Zhang Q, Zhang K, Hu G (2016) Smart three-dimensional lightweight structure triggered from a thin composite sheet via 3D printing technique. Sci Rep. https://doi.org/10.1038/srep22431

16. Gladman AS, Matsumoto EA, Nuzzo RG, Mahadevan L, Lewis JA (2016) Biomimetic 4D printing. Nat Mater 15(4):413-418

17. Bakarich SE, Gorkin R, Spinks GM (2015) 4D printing with mechanically robust, thermally actuating hydrogels. Macromol Rapid Commun 36(12):1211-1217

18. Kuksenok O, Balazs AC (2016) Stimuli-responsive behaviour of composites integrating thermo-responsive gels with photoresponsive fibers. Mater Horiz 3(1):53-62

19. Pei E (2014) 4D printing: dawn of an emerging technology cycle. Assem Autom 34(4):310-314

20. Pei E (2014) 4D printing - revolution or fad? Assem Autom 34(2):253-244

21. Monzon MD, Paz R, Pei E, Ortega F, Suarez LA, Ortega Z, Aleman ME, Plucinski T, Clow N (2016) 4D printing: processability and measurement of recovery force in shape memory polymers. Int J Adv Manuf Technol 89(5-8):1827-1836

22. Zhou Y, Hwang WM, Kang SF, Wu XL, Lu HB, Fu J, Cui H (2015) From 3D to 4D printing: approaches and typical applications. J Mech Sci Technol 29(10):4281-4288

23. Tibbits S, Papadopoulou A, McKnelly C, Martin C, Campos F (2013) Programmable materials. Self-Assembly Lab, Christophe Guberan, Erik Demaine, Carbitex LLC, Autodesk Inc. http:// www.selfassemblylab.net/ProgrammableMaterials.php. Accessed 24 Nov 2017

24. Campbell TA, Tibbits S, Garrett B (2014) The programmable world. Sci Am 311:60-65

25. Taylor DL (2016) Self-healing hydrogels. Adv Mater 28(41):9060-9093

26. Frost and Sullivan (2014) 4-D printing to usher in age of lowlabour, fast-paced product manufacturing. http://images.discover. frost.com/Web/FrostSullivan/NA_PR_ABrown_D545-TI_21Aug 2014.pdf. Accessed 24 Nov 2017

27. Patil D, Song G (2017) A review of shape memory material's applications in the offshore oil and gas industry. Smart Mater Struct 26:093002 
28. Sun L, Huang WM, Ding Z, Zhao Y, Wang CC, Purnawali H, Tang C (2012) Stimulus-responsive shape memory materials: a review. Mater Des 33:577-640

29. Zhou J, Sheiko SS (2016) Reversible shape-shifting in polymeric materials. J Polym Sci Part BPolym Phys 54:1365-1380

30. Erkeçoğlu S, Sezer AD, Bucak S (2016) Smart delivery systems with shape memory and self-folding polymers. "Smart Drug Delivery System”, book edited by Ali Demir Sezer (ISBN 978-953-51-2247-0)

31. Liu C, Qin H, Mather PT (2007) Review of progress in shapememory polymers. J Mater Chem 16:1543-1558

32. Yang WG, Lu H, Huang WM, Qi HJ, Wu XL, Sun KY (2014) Advanced shape memory technology to reshape product design, manufacturing and recycling. Polymers 6:2287-2308

33. Lendlein A, Kelch S (2002) Shape memory polymers. Angew Chem Int Ed 41(12):2034-2057

34. Behl M, Razzaq MR, Lendlein A (2010) Multifunctional shapememory polymers. Adv Mater 22:3388-3410

35. Zhou J, Turner SA, Brosnan SM, Li Q, Cartillo JY, Nykypanchuk D, Gang O, Ashby VS, Dobrynin AV, Sheiko SS (2014) Shapeshifting: reversible shape memory in semicrystalline elastomers. Macromolecules 47:1768-1776

36. Basit A, L'Hostis G, Pae MJ, Durand B (2013) Thermally activated composite with two-way and multi-shape memory effects. Materials 6:4031-4045

37. Chen S, Hu J, Zhuo H, Zhu Y (2008) Two-way shape memory effect in polymer laminates. Mater Lett 62:4088-4090

38. Bellin I, Kelch S, Langer R, Lendlein A (2006) Polymeric tripleshape materials. Proc Natl Acad Sci 103(48):18043-18047

39. Bothe M, Mya KY, Lin EMJ, Yeo CC, Lu X, He C, Pretsch T (2012) Triple-shape properties of star-shaped POSS-polycaprolactone polyurethane networks. Soft Matter 8(4):965-972

40. Li X, Zhu Y, Dong Y, Fu Y (2015) Epoxy resin composite bilayers with triple-shape memory effect. J Nanomater 2015:475316

41. Antonelli P (2008) Design and elastic mind, 01 edn. The Museum of Modern Art, New York

42. Maxey K (2017) Sequentially self-folding structures activated by light. http://www.engineering.com/DesignerEdge/DesignerEd geArticles/ArticleID/14461/Sequentially-Self-Folding-Structures -Activated-by-Light.aspx. Accessed 24 Nov 2017
43. Peraza-Hernandez EA, Hartl DJ, Malak RJ Jr, Lagoudas DC (2014) Origami-inspired active structures: a synthesis and review. Smart Mater Struct. 23(9):094001

44. Peraza-Hernandez EA, Hu S, Kung HW, Hartl D, Akleman E (2013) Towards building smart self-folding structures. Comput Graph 37(6):730-742

45. Pei E, Loh GH, Harrison D, Almeida HDA, Monzon MD, Paz R (2017) A study of 4D printing and functionally graded additive manufacturing. Assem Autom 37(2):147-153

46. Loh GH (2017) Building a conceptual understanding of functionally graded additive manufacturing (FGAM) and its limitation. In: 15th rapid design, prototyping and manufacturing conference. Northumbria University, 28th April 2017

47. Sethna JP (2017) Digital material. http://www.lassp.cornell.edu/ sethna/DM/. Accessed 24 Nov 2017

48. Janbaz S, Hedayati R, Zadpoor AA (2016) Programming the shape-shifting of flat soft matter: from self-rolling/self-twisting materials to self-folding origami. Mater Horiz 3:536-547

49. Demaine ED (2001) Folding and unfolding linkages, paper, and polyhedra. Discrete and computational geometry. Springer, Berlin, pp 113-124

50. Ryu J, D’Amato M, Cui X, Long KN, Qi HJ, Dunn ML (2012) Photo-origami-bending and folding polymers with light. Appl Phys Lett 100(2012):161908

51. Ionov L (2013) 3D microfabrication using stimuli-responsive selffolding polymer films. Polym Rev 53:92-107

52. Ionov L (2010) Actively-moving materials based on stimuliresponsive polymers. J Mater Chem 20:3382-3390

53. Liu Y, Genzer J, Dickey MD (2016) “2D or not 2D”: shape-programming polymer sheets. Prog Polym Sci 52(2016):79-106

54. Gracias DH (2013) Stimuli responsive self-folding using thin polymer films. Curr Opin Chem Eng 2:112-119

Publisher's Note Springer Nature remains neutral with regard to jurisdictional claims in published maps and institutional affiliations. 